\title{
Asynchronous Cooperative Diversity
}

\author{
Shuangqing Wei, Dennis L. Goeckel, and Matthew C. Valenti
}

\begin{abstract}
Cooperative diversity, which employs multiple nodes for the simultaneous relaying of a given packet in wireless ad hoc networks, has been shown to be an effective means of improving diversity, and, hence, mitigating the detrimental effects of multipath fading. However, in previously proposed cooperative diversity schemes, it has been assumed that coordination among the relays allows for accurate symbol-level timing synchronization at the destination and orthogonal channel allocation, which can be quite costly in terms of signaling overhead in mobile ad hoc networks, which are often defined by their lack of a fixed infrastructure and the difficulty of centralized control. In this paper, cooperative diversity schemes are considered that do not require symbol-level timing synchronization or orthogonal channelization between the relays employed. In the process, a novel minimum mean-squared error (MMSE) receiver is designed for combining disparate inputs in the multiple-relay channel. Outage probability calculations and simulation results demonstrate the not unexpected significant performance gains of the proposed schemes over single-hop transmission, and, more importantly, demonstrate performance comparable to schemes requiring accurate symbol-level synchronization and orthogonal channelization.
\end{abstract}

Index Terms-Cooperative diversity, asynchronous wireless network, minimum mean-squared error (MMSE) receiver, outage probability.

\section{INTRODUCTION}

D RIVEN by a strong demand for personal communications systems and devices, the wireless communications market has undergone tremendous growth in the past few decades. As an alternative to cellular networks, ad hoc wireless networks have attracted considerable attention in recent years. An ad hoc wireless network is a collection of wireless mobile nodes that self-configure to form a network without the aid of any established infrastructure [1]. Thus, unlike cellular systems, in which the coordination of mobiles in a given area is done via the basestations, ad hoc networks utilize other mobiles as relays to transfer information from a source to its destination. The lack of required infrastructure in an ad

Manuscript received May 17, 2004; revised September 26, 2005; accepted January 11, 2006. The associate editor coordinating the review of this letter and approving it for publication was H. Yanikomeroglu. This paper was presented in part at the Conference on Information Sciences and Systems (CISS), Princeton, March 2004. Dennis Goeckel was supported in part by the National Science Foundation under CAREER Award No. CCR-9875482, and Grants EIA-0080119, ECS-0300130 and CCF-0430892. Shuangqing Wei was supported in part by the Board of Regents of Louisiana under grants LEQSF(2004-07)-RD-A-17 and NSF/LEQSF(2005)-PFUND-10.

Shuangqing Wei is with the Department of ECE, Louisiana State University, Baton Rouge, LA 70802 (e-mail: \{swei\} @ece.lsu.edu).

Dennis L. Goeckel is with the Electrical and Computer Engineering Department, University of Massachusetts, Amherst, MA 01003 (e-mail: \{goeckel\}@ecs.umass.edu).

Matthew C. Valenti are with the Lane Department of Computer Science and Electrical Engineering, West Virginia University, Morgantown, WV 265066109 (e-mail: \{mvalenti\}@wvu.edu).

Digital Object Identifier 10.1109/TWC.2006.04332.

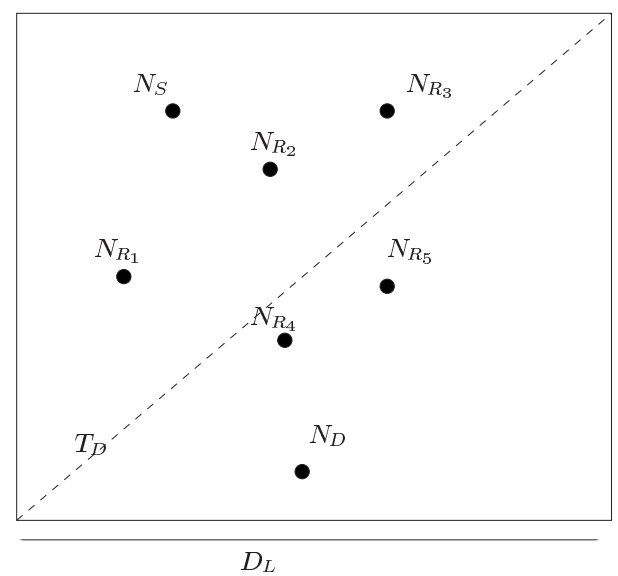

Fig. 1. Ad hoc wireless network. Node $N_{S}$ wishes to communicate with node $N_{D}$ with the help of relay nodes $N_{R_{1}}, N_{R_{2}} \cdots, N_{R_{5}}$ in a region of size $D_{L} \times D_{L}$ with maximum signal propagation delay $T_{D}$.

hoc wireless network is highly appealing in both military and commercial applications [1].

The impairments caused by multipath fading and the timevarying nature of the wireless channel must be considered in designing an ad hoc wireless network. The broadcast nature of the radio channel introduces characteristics in ad hoc wireless networks that can be exploited in the form of cooperative diversity [2], a strategy whereby cooperating nodes between the source and its destination forward the received data generated by the source to the destination after some processing at each relay terminal as shown in Fig. 1.

In [3], [4], the authors developed and analyzed several energy-efficient cooperative diversity protocols that combat fading in wireless networks. In their work, different nodes in the wireless network share their antennas and resources to create a virtual array. For the decode-and-forward scheme proposed in this work, the relay nodes first fully decode the transmission from the source terminal. Next, they will either forward that information to the destination in the assigned slot, or they can cooperatively utilize a space-time code to allow the destination terminal to take advantage of distributed spatial diversity to average out the fading. In [5], [6], [7], to achieve a better trade-off between energy and spectral efficiency while using similar orthogonal channel allocation schemes as in [3], various coding schemes were proposed across the source and the relay nodes, which achieve not only cooperative diversity gain but also coding gain. The aforementioned cooperative schemes assume orthogonal channel allocation and synchronization of the signals of the cooperating terminals at the receiver, both of which require significant overhead in an ad hoc wireless network.

To the authors' knowledge, [8] is the only paper that deals 
with relaying when orthogonal channel allocation is impossible in an ad hoc wireless network. In [8], they essentially employ adaptive decode and forward or amplify and forward schemes (i.e non-regenerative relaying schemes [9], [10]) on each relay node as in [11], so that relay nodes can choose to transmit or remain silent depending on the received signal-tonoise-ratio (SNR). The main difference between the solution described here and that of [8] is that the scheme of [8] is limited in efficiency by the need to intentionally increase the data symbol period $T_{s}$ to the avoid the inter-symbolinterference (ISI) caused by the asynchronous transmission of signals to the destination. In contrast, here we designs a novel receiver to avoid such a penalty. Additionally, we also demonstrate that artificially introducing delays at the relays can significantly improve system performance.

As seen from the above, the existing cooperative diversity schemes [3], [4], [5], [6], [7], [12] are more suitable for cellular architectures because orthogonal channel allocation and/or synchronization of relay nodes are assumed, which will be difficult in ad-hoc wireless networks due to their infrastructureless nature. Therefore, finding schemes which relax this coordination is important for achieving cooperative diversity in practice. In this paper, this problem will be attacked with approaches that capture the essence of ad hoc networks from a physical layer design perspective. In particular, a distributed delay diversity approach is proposed to achieve the diversity gains promised by distributed spacetime codes. Unlike the extension of other approaches [13] to the synchronization problem in distributed space-time coding, the proposed system also admits a robust and easily trainable receiver when synchronization is not present in the system.

For a cellular network, a transmit diversity scheme was proposed in [14] that had multiple antennas at the basestation transmit various delayed versions of a common signal to create an effectively frequency-selective fading channel at a single antenna at the mobile receiver. When there are multiple antennas at one base station, transmitting various delayed versions of the same signal on the transmit antennas can be easily implemented. However, in the case of an ad hoc network with multiple relays, the recovered signals at each relay node are not necessarily identical due to the demodulation/decoding errors resulting from the presence of fading and thermal noise on the link from the source to the relay. To address this, a cyclic redundancy check (CRC) code [15] can be used so that each relay will know if it received a correct packet. If the received packet is error-free, the relay node will then forward the information packet to the destination, after possibly introducing an additional intentional artificial delay (see Section II-B). If not, this packet of data will be dropped by the relay. Assuming that the CRC code enables the relay nodes to correctly detect all packet errors, the destination node will see an equivalent multipath fading channel in the form of the artificially introduced relay delays.

Per the above, this manuscript contributes to the development of realizable solutions for providing cooperative diversity in wireless ad hoc networks. There are two main contributions: (1) two related protocols that implement distributed delay diversity; (2) the development of a novel fractional spaced decision feedback equalizer (DFE) that combines the inputs

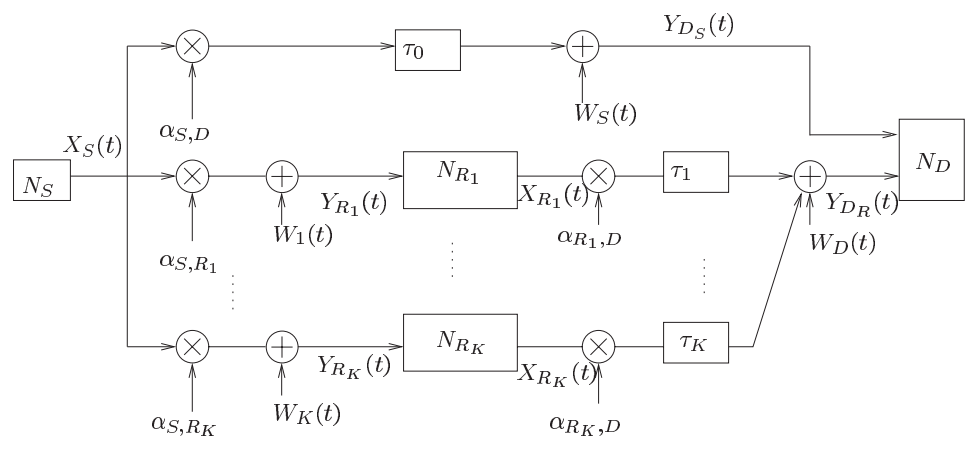

Fig. 2. System model of an ad hoc wireless network under the assumption that all $\mathrm{K}$ relays are in the decoding set.

from two independent channels, one that is frequency selective (from the relays), and a second that is frequency nonselective (directly from the source), and minimizes the mean squared error (MMSE) at the decision point in the receiver

\section{Proposed PRotocols}

\section{A. System Model}

Consider an ad hoc network as shown in Fig.1, where the source node $N_{S}$ transmits messages to the destination node $N_{D}$ with the help of a set of $K$ nearby relays $\mathcal{R}=$ $\left\{N_{R_{1}}, N_{R_{2}}, \ldots, N_{R_{K}}\right\}$. Relays are indexed according to their distance from the source, with $N_{R_{1}}$ being closest to the source and $N_{R_{K}}$ being the furthest. Each node has a single halfduplex radio and a single antenna. Data is formatted into packets and each packet is protected by a CRC error detecting code, which we assume is always able to detect a packet error.

In this paper, we mainly focus on a two-hop scenario whose mathematical model is shown in Fig. 2, though the results can be generalized for the multi-hop case. In the two-hop case, signaling of the packet occurs over two orthogonal time slots, and it is assumed that the inter-node distances are fixed for the duration of both time slots. During the first slot, termed the broadcast period, the source broadcasts to both $N_{D}$ and a set $\mathcal{P} \subseteq \mathcal{R}$ of the relays. The relays in $\mathcal{P}$ are the participating relays. Each relay $N_{R_{k}} \in \mathcal{P}$ receives a signal $Y_{R_{k}}(t)$ from the source and, after demodulation, checks for errors using the CRC code. The set $\mathcal{D} \subseteq \mathcal{P}$ of participating relays that receive the packet without errors is called the decoding set, and the number of decoding relays $|\mathcal{D}|=M$. The value of $M$ is random and depends on the channel quality between source and each relay.

During the second time slot, termed the relay period, all nodes in $\mathcal{D}$ transmit signals $X_{R_{k}}(t)$ to $N_{D}$, which combines the transmissions from the relay(s) and a stored copy of the source transmission using the joint DFE receiver described in Section III. In Fig. 2, it is assumed that all $K$ relays are in $\mathcal{D}$, but in general some relays could detect errors and not participate in the relay period.

Narrow-band transmission is assumed here, where the channel between any pair of nodes is frequency non-selective. In addition, quasi-static fading is assumed, where the path gains remain fixed during the transmission of a whole packet, but are independent from node to node and packet to packet. Time 
delays are introduced on each path from the source to the destination. This time delay incorporates the processing time at the relay nodes and the propagation delay of the whole route. More specifically, $\tau_{0}$ is the delay from $N_{S}$ to $N_{D}$, and $\tau_{k}$ is the cumulative delay for the transmission from $N_{S}$ to $N_{R_{k}}$, processing at $N_{R_{k}}$ and for transmission from $N_{R_{k}}$ to $N_{D}$. The noise processes $W_{S}(t), W_{D}(t)$ and $W_{k}(t), k=1, \cdots, K$ are independent complex white Gaussian noise with two-sided power spectral density $\mathcal{N}_{0}$. The complex channel gain $\alpha_{i, j}$ captures the effects of both path loss and the quasi-static fading from node $N_{i}$ to node $N_{j}$, where $i \in\left\{S, R_{1}, \cdots, R_{K}\right\}$, and $j \in\left\{R_{1}, \cdots, R_{K}, D\right\}$. Statistically, $\alpha_{i, j}$ will be modeled as zero mean, mutually independent complex jointly Gaussian random variables with variances $\sigma_{i, j}^{2}$. The fading variances can be assigned using wireless path loss models based on the network geometry. Here, it is assumed that $\sigma_{i, j}^{2} \propto 1 / d_{i, j}^{\mu}$, where $d_{i, j}$ is the distance from node $N_{i}$ to $N_{j}$, and $\mu$ is a constant whose value, as estimated from field experiments, lies in the range $2 \leq \mu \leq 5$ [16]. It is assumed that $\alpha_{i, j}$ is estimated accurately at the receiver, but is not available to the transmitter. Likewise, since significant higher-layer coordination is undesirable per Section I, it is assumed that each relay node transmits with identical power unless otherwise stated.

A practical constraint prohibiting the relay nodes from transmitting and receiving at the same time [17] will be assumed, which results in orthogonality in time between the packet arriving at $N_{D}$ via the direct path from $N_{s}$ and the collection of packets arriving at $N_{D}$ through relay nodes. Note that orthogonality between the signals $X_{R_{1}}(t), X_{R_{2}}(t), \cdots, X_{R_{K}}(t)$ transmitted from different relay nodes, $N_{R_{1}}, N_{R_{2}}, \cdots, N_{R_{K}}$, is not assumed and forms the crux of the problem. The difference $\tau_{k}-\tau_{0}$ includes the processing time of a whole packet at $N_{R_{k}}$ in addition to the relative propagation delay between the $k$ th relay path and the direct path. Without loss of generality, $\tau_{0}$ is set to zero. Under the modelling assumed above, the signals in Fig. 2 are: $Y_{R_{k}}(t)=\alpha_{S, R_{k}} X_{S}(t)+W_{k}(t), k=$ $1, \cdots, K, Y_{D_{s}}(t)=\alpha_{S, D} X_{S}(t)+W_{S}(t)$, and $Y_{D_{R}}(t)=$ $\sum_{j=1}^{K} \alpha_{R_{j}, D} X_{R_{j}}\left(t-\tau_{j}\right)+W_{D}(t)$, where $Y_{D_{s}}(t)$ and $Y_{D_{R}}(t)$ have no common support in the time domain, and $W_{S}(t)$ and $W_{D}(t)$ are independent and identically distributed white Gaussian random processes. The transmitted signal from the source is $X_{S}(t)=\sum_{k=-\infty}^{\infty} I_{k} h_{T x}(t-k T)$, where $h_{T x}(t)$ is the impulse response of the transmit pulse shaping filter, $T$ is the symbol period, and $I_{k}$ is the $k$ th complex data symbol with $I_{k}=a_{k}+j b_{k}, \frac{1}{2} E\left[\left|I_{k}\right|^{2}\right]=\sigma_{I}^{2}=1$, and $\left\{I_{k}\right\}$ is a sequence of uncorrelated symbols that is independent of $\left\{W_{j}(t)\right\}$. It is assumed that $h_{T x}(t)$ has a squared root raised cosine (SRRC) impulse response with a filter roll-off factor $\beta \in[0,1]$. Denote each front-end receiver filter response as $h_{R x}(t)$, which is matched to the SRRC transmitter filter, i.e., $h_{R x}(t)=h_{T x}(-t)$. It is assumed that all nodes transmit with equal power $P=\sigma_{I}^{2}$.

Because outage probability is a more suitable metric than average error probability for quasi-static fading, the measure of performance is the outage probability of the frame error rate (FER), which is defined as follows. Given one realization of the connection between the source node and the destination node (i.e., one realization of the random locations of $K+2$ nodes in a given area, realizations of channel fading gains $\alpha_{i, j}$ and the set $\mathcal{D}$ ), the frame error event at $N_{D}$ is caused by the additive noise and ISI in the equivalent multipath channel. An outage is declared when the FER at $N_{D}$ is above a predetermined threshold (e.g., 0.1). The outage probability will be obtained by then calculating the likelihood of such an event over the realizations of the random connection between node $N_{S}$ and $N_{D}$.

\section{B. Protocol Description}

Two protocols are proposed, which differ in the composition of $\mathcal{P}$ :

Closest Relays Participate (CRP): In this protocol, the participating set is $\mathcal{P}=\left\{N_{R_{1}}, N_{R_{2}}\right\}$, i.e. only the two relays that are closest to the source participate. While this protocol serves to illustrate key concepts associated with the proposed system, its actual implementation would require the determination of which two nodes are closest to the source, which imposes additional overhead on the system. However, as the number of possible relay nodes per-hop in this protocol is upperbounded, if a system can meet the demand of such overhead in selecting the closest two relay nodes, CRP is a viable option which costs less overall energy across network than the ARP protocol described below.

The motivation for employing the two nodes closest to the source in the CRP protocol is to maximize the likelihood that the relay-destination link is able to achieve diversity two. The CRP protocol is largely included to demonstrate key aspects of the idea, whereas the protocol described next is shown to be more effective in simulation.

All Relays Participate (ARP): In this protocol, all relays in the network participate, and so $\mathcal{P}=\mathcal{R}$. The advantage of this protocol is that there is very little centralized control required, since any node that decodes a packet heard during the broadcast period simply transmits during the relay period. On the other hand, this protocol might cause many nodes involved in forwarding source information, which results in more energy consumption across network then the CRP protocol. One way to handle this is to normalize the transmission power by dividing the relay transmission power by $M$. However, this number is not known a priori and must be estimated.

Each relay can either transmit its signal immediately after processing or after an artificial delay, which gives rise to two versions of the protocol:

No Delay (ND): Each relay in $\mathcal{D}$ transmits the signal immediately after receiving and processing the packet (we assume the processing time is the same for all relays). As shown in the numerical results, the performance under this protocol can be limited if the overall path delays of the relay nodes are similar, and, hence, the anticipated delay diversity is not always achieved.

Artificial Delay (AD): Each relay in $\mathcal{D}$ waits for a random amount of time. The delay timer is selected from a pool of possible artificial delays (e.g. $\{T, 2 T, \ldots\}$ ). Before a node transmits during the relay period, it delays the signal by its currently assigned artificial delay. Numerical results will demonstrate that by choosing the pool carefully and assigning different artificial delays to nodes in close geographical prox- 
imity, significant performance gains are observed, because the desired delay diversity is almost always achieved.

Altogether, there are four options for the protocol, which are denoted CRP/ND, CRP/AD, ARP/ND, and ARP/AD.

The pool of possible artificial delays used by variant AD is a key system design issue. For pools containing only short delays, receiver implementation will be simpler because the effective ISI channel will be shorter, but there will be a higher chance of encountering node geometries that incur diversity loss. Conversely, for longer delays, receiver implementation is more difficult, but there is a less chance of such diversity loss (as shown in Section 3.2.2). Throughout this paper, a pool $\{T, 2 T, \ldots\}$ will be employed. This provides a realizable receiver as described in Sections III and IV, while providing sufficient resolvable delay to help achieve diversity. In addition, the induced delay, which is on the order of symbol periods, is not significant relative to the packet length.

In the simulation results, it will be assumed that the allotment of artificial delays from the pool to the relay nodes is done randomly. However, it is certainly possible to envision simple medium access control (MAC) protocols that improve such an assignment, resulting in nodes in close geographical proximity employing different delays from the pool. Although we avoid complete specification of a MAC protocol to accomplish such, a general idea is described here to demonstrate that near-optimal assignment should be possible. Consider an ad hoc wireless network employing the ARP protocol, where each node carries with it a current artificial delay from the pool that it will employ whenever it is asked to serve as a relay. Now, whenever a source transmits a packet, it attaches at the end of that packet a few bits indicating the artificial delay from the pool it would employ if it were playing the role of relay. Per above, all participating relays hear the transmission and attempt decoding. If a node is successful, which is more likely if the node is near the source, and it realizes the source is employing the same artificial delay as itself, the node switches randomly to a different artificial delay from the pool. Although this does not impact performance for the current packet transmission, since the source will not transmit during the relay period, future transmissions where the geographically-close current source and current relay might both be asked to serve as relays, will be improved. In effect, this protocol encourages the desired condition - that nodes in close proximity are assigned different artificial delays from the pool - and will work to maintain such even under node mobility.

\section{JOINT DFE RECEIVER}

The proposed system requires a receiver that can jointly process the output of a frequency-selective channel and the output of an independent frequency-nonselective channel. For the application considered here, these channels correspond to that from the relays and that from the source, respectively. A straightforward approach would be to first employ a standard equalizer to process the signal from the frequency-selective channel; then, a number of straightforward combining schemes could be used to combine the output of such with the signal from the orthogonal channel. However, it will be shown here

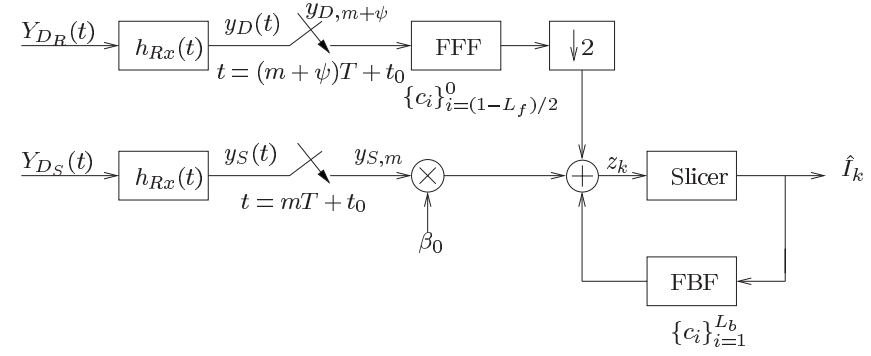

Fig. 3. Functional description of the joint DFE receiver. The signal $Y_{D_{s}}(t)$, and hence $y_{S, k}$, is collected by the receiver while the source is transmitting, and the signal $Y_{D_{R}}(t)$, and hence $y_{D, k+\psi}$, is collected by the receiver while the relays are transmitting, and then both are processed jointly after the signal from the source is delayed to coincide with the relayed signal.

that such schemes are inferior to an equalizer specifically designed to jointly process the output of the two channels under the MMSE criterion. Hence, in this section, the joint DFE receiver structure is motivated, and the coefficients of the DFE feedforward filter (FFF), DFE feedback filter (FBF), and channel combining are chosen jointly based on an MMSE criterion. Note that this receiver structure will be more broadly applicable, as it can be employed in any application where multiple orthogonal channels, some of which are frequency selective, need to be jointly processed. For example, its extension to the case that some node-to-node channels are inherently frequency-selective is straightforward.

A major advantage of the delay diversity approach proposed here over more complicated space-time coding strategies [13] is that the vast literature on adaptive equalization can be exploited to develop rapidly trainable receivers. In particular, even though not considered in the present paper, the receiver need not know anything about the number of relays involved in a transmission, the real or artificial delays for a given relay transmission, or the fading parameters. Instead, it can find the taps of its filters and the combining coefficient by simply driving a standard adaptive equalization algorithm either blindly or based on a known training sequence (identical across all relays) transmitted at the start of any relay transmission.

\section{A. Receiver and Optimization}

Let $h_{c, R}(t)=\sum_{i \in \mathcal{D}} \alpha_{i, D} \delta\left(t-\tau_{i}\right)$ be the complex baseband impulse response of the equivalent multipath channel between the collection of transmitting relays and the destination node. Denote the raised cosine (RC) pulse by $h_{R C}(t)=$ $h_{T x}(t) \otimes h_{T x}(-t)$, where $\otimes$ is the convolution operation. Thus the equivalent complex baseband impulse response at the destination for the transmissions from the relays is $h_{D}(t)=$ $\sum_{i \in \mathcal{D}} \alpha_{i, D} h_{R C}\left(t-\tau_{i}\right)$. Per Section II-A it will be assumed that the receiver is able to accurately estimate this channel. The complex noise at the output of the receiver filter is $\nu_{D}(t)=W_{D}(t) \otimes h_{R x}(t)$, which has autocorrelation function $\phi_{\nu_{D}}(\tau)=\frac{1}{2} E\left[\nu_{D}(t) \nu_{D}^{*}(t+\tau)\right]=\mathcal{N}_{0} h_{R C}(\tau)$ for the SRRC receiver. The receiver structure is depicted in Fig. 3. Note the difference from the standard DFE, since the signal $Y_{D_{S}}(t)$ received while the source is transmitting must also be jointly processed. This structure, along with the joint optimization of 
such, is one of the contributions of this work. The continuoustime signal at the output of $h_{R x}(t)$ at $N_{D}$ during the time the relays are transmitting is $y_{D}(t)=h_{R x}(t) \otimes Y_{D_{R}}(t)=$ $\sum_{n=-\infty}^{\infty} I_{n} h_{D}(t-n T)+\nu_{D}(t)$.

The fractional spaced (i.e., T/2 spaced) equalizer is considered due to its robust performance. Similar notation to [18] will be employed. The input-output relation for the discretetime equivalent channel from the multiple relays to the input of the feedforward filter at the destination is $y_{D, k+\psi} \triangleq$ $y_{D}\left[(k+\psi) T+t_{0}\right]=\sum_{n=0}^{L} I_{k-n} h_{D, n+\psi}+\nu_{D, k+\psi}$, where $h_{D, n+\psi}=h_{D}\left((n+\psi) T+t_{0}\right), \nu_{D, k+\psi}=\nu_{D}\left(k T+\psi T+t_{0}\right)$ with $k$ and $n$ integer (int.), and $\psi \in\left\{0, \frac{1}{2}\right\}$. By properly selecting the initial sampling time $t_{0}$, the channel impulse response $h_{D}\left(t+t_{0}\right)$ is approximated as nonzero over the time interval $[0, L T]$, where $L$ is an integer. The FFF is an anti-causal filter with $L_{f} T / 2$-spaced taps and coefficients $\left\{c_{\left(1-L_{f}\right) / 2}, \cdots, c_{-1}, c_{-1 / 2}, c_{0}\right\}$. The FBF is a causal filter with $L_{b} T$-spaced taps and coefficients $\left\{c_{1}, c_{2}, \cdots, c_{L_{b}}\right\}$. The length $L_{b}$ of the FBF is assumed to be equal to the length of the channel, i.e., $L_{b}=L$ [19]. In practice, $L_{f}$ is chosen one to five times the channel pre-cursor length, which is determined by the position of the peak amplitude response of $h_{D}\left(t+t_{0}\right)$ [19].

For the signal $Y_{D_{S}}(t)$ received while the source node is transmitting, whose support does not overlap that of $Y_{D_{R}}(t)$ per Section II-A, the equivalent discrete-time channel model at the output of the T-spaced sampler is

$$
y_{S, k}=y_{S}\left(k T+t_{0}\right)=\alpha_{S, D} I_{k}+\nu_{S, k},
$$

where $y_{S}(t)$ is the output of the receiver filter during the time the source is transmitting and $\left\{\nu_{S, k}\right\}$ is a sequence of independent complex Gaussian random variables with zero mean and variance $E\left[\left|\nu_{S, k}\right|^{2}\right]=2 \mathcal{N}_{0}$ which are independent of $\left\{\nu_{D, k+\psi}\right\}$.

The coefficients of the FFF and FBF, as well as $\beta_{0}$ which is used to scale and derotate for the direct path $y_{S, k}$, can be obtained by assuming correct past decisions and minimizing the mean squared error (MSE) $\mathrm{MSE}=E\left[\left|I_{k}-\mathbf{u}_{k}^{T} \mathbf{c}\right|^{2}\right]$, where the $\mathrm{x}^{T}$ denotes non-conjugate transpose of a vector $\mathbf{x}$. The data vector $\mathbf{u}_{k}$ is defined as

$$
\begin{aligned}
\mathbf{u}_{k} \triangleq \quad & {\left[y_{D, k+\left(L_{f}-1\right) / 2}, \cdots, y_{D, k+1}, y_{D, k+1 / 2},\right.} \\
& \left.y_{D, k}, y_{S, k}, I_{k-1}, I_{k-2}, \cdots, I_{k-L_{b}}\right]^{T},
\end{aligned}
$$

and the vector of filter coefficients $\mathbf{c}$ is denoted as

$$
\begin{aligned}
\mathbf{c} \triangleq & {\left[c_{\left(1-L_{f}\right) / 2}, \cdots, c_{-1}, c_{-1 / 2}, c_{0},\right.} \\
& \left.\beta_{0}, c_{1}, c_{2}, \cdots, c_{L_{b}}\right]^{T} .
\end{aligned}
$$

By applying the orthogonality principle [20], i.e., $E\left[\left(I_{k}-z_{k}\right) \mathbf{u}_{k}^{*}\right]=0$, where $z_{k}=\mathbf{u}_{k}^{T} \mathbf{c}$, the filter coefficients are determined as (please see the Appendix A for the detailed derivations):

$$
\begin{gathered}
\mathbf{c}_{\left(1-L_{f}\right) / 2: 0}=\left(1-\beta_{0} \alpha_{S, D}\right) \cdot \Omega^{-1} \mathbf{p} \\
\beta_{0}=\alpha_{S, D}^{*} \frac{1-\tilde{U}_{0}}{2 \mathcal{N}_{0} / \sigma_{c}^{2}+\left|\alpha_{S, D}\right|^{2}\left(1-\tilde{U}_{0}\right)},
\end{gathered}
$$

and

$$
c_{j}=-\sum_{i=\left(1-L_{f}\right) / 2: 0} h_{D, j-i} \cdot c_{i}, j=1,2, \cdots, L_{b},
$$

where $\left(1-L_{f}\right) / 2: 0=\left(1-L_{f}\right) / 2, \cdots,-1,-1 / 2,0, \mathbf{p}$ is a column vector of dimension $L_{f}$ with $\mathbf{p}_{i}=h_{D,-i}^{*}, i=$ $\left(1-L_{f}\right) / 2: 0$, and the scalar $\tilde{U}_{0}=\mathbf{p}^{\dagger} \mathbf{\Omega}^{-1} \mathbf{p}$, where $\mathbf{p}^{\dagger}$ is the conjugate transpose of $\mathrm{p}$. The matrix $\Omega$ is determined by the autocorrelation of the data vector $\mathbf{u}_{k}, \boldsymbol{\Omega}=\boldsymbol{\Gamma}-\boldsymbol{\Lambda}^{*} \boldsymbol{\Lambda}^{T}$, where the elements of matrices $\boldsymbol{\Gamma}$ and $\boldsymbol{\Lambda}$ are

$$
\begin{aligned}
\boldsymbol{\Gamma}_{i, j} & =\frac{1}{2} E\left[y_{D, k-i} y_{D, k-j}^{*}\right] \\
& =\sum_{n \in \mathcal{Z}} h_{D, n-i}^{*} h_{D, n-j}+\phi_{\nu_{D}}((i-j) T)
\end{aligned}
$$

for $i, j \in\left\{\frac{1-L_{f}}{2}, \cdots,-1,-1 / 2,0\right\}$, and

$$
\boldsymbol{\Lambda}_{i, j}^{T}=h_{D, i-j}
$$

for $i \in\left\{1,2, \cdots, L_{b}\right\}$, and $j \in\left\{\left(1-L_{f}\right) / 2: 0\right\}$.

\section{B. Performance Analysis}

1) Mean Squared Error: To aid in the performance analysis, suppose temporarily that only the combined relay signal $Y_{D_{R}}(t)$ is used to estimate the source packet $\left\{I_{k}\right\}$, i.e., $\left.Y_{D_{S}}(t)\right)$ is disregarded. The coefficient vector $\tilde{\mathbf{c}}$ of the FFF and FBF has been obtained in [21], [18] using the MMSE criterion, which is denoted as $\tilde{\mathbf{c}} \triangleq$ $\left[\tilde{c}_{\left(1-L_{f}\right) / 2}, \cdots, \tilde{c}_{-1}, \tilde{c}_{-1 / 2}, \tilde{c}_{0}, \tilde{c}_{1}, \tilde{c}_{2}, \cdots, \tilde{c}_{L_{b}}\right]^{T}$, with entries $\tilde{\mathbf{c}}_{\left(1-L_{f}\right) / 2: 0}=\boldsymbol{\Omega}^{-1} \mathbf{p}$, and $\tilde{c}_{j}=-\sum_{i=\left(1-L_{f}\right) / 2: 0} h_{D, j-i}$. $\tilde{c}_{i}, j=1,2, \cdots, L_{b}$. Given $\tilde{\mathbf{c}}$, the minimum mean squared error for the standard DFE can be shown to be $(\mathrm{MSE})_{0}=$ $\sigma_{c}^{2}\left(1-\tilde{U}_{0}\right)$ [22], where $\sigma_{c}^{2}=2 \sigma_{I}^{2}=2 s$.

However, when $Y_{D_{S}}(t)$ and $Y_{D_{R}}(t)$ are employed to jointly estimate $I_{k}$, the minimum mean squared error can be shown to be (see Appendix A):

$$
(\mathrm{MSE})_{o}=(\mathrm{MSE})_{o} \frac{2 \mathcal{N}_{0}}{2 \mathcal{N}_{0}+\left|\alpha_{S, D}\right|^{2}(\mathrm{MSE})_{o}} .
$$

The input to the slicer $z_{k}$ can be expressed in a generic way, i.e., $z_{k}=\mathbf{u}_{k}^{T} \mathbf{c}=I_{k} U_{0}+\sum_{n \neq 0} I_{k-n} U_{n}+\tilde{\nu}_{k}$, where $\tilde{\nu}_{k}$ is Gaussian noise term which is independent of the ISI, and $\left\{U_{n}\right\}$ are ISI coefficients. The signal-to-interference-and-noise-ratio (SINR) at the input of the slicer is thus defined as [22]

$$
\operatorname{SINR}=\frac{\left|U_{0}\right|^{2} \sigma_{c}^{2}}{\sigma_{c}^{2} \sum_{n \neq 0}\left|U_{n}\right|^{2}+E\left|\tilde{\nu}_{k}\right|^{2}}
$$

Combining Eq. (10) and Eq. (9), we obtain

$$
\mathrm{SINR}=\frac{\sigma_{c}^{2}-(\mathrm{MSE})_{o}}{(\mathrm{MSE})_{o}}=\operatorname{SINR}+\frac{\sigma_{c}^{2}\left|\alpha_{S, D}\right|^{2}}{2 \mathcal{N}_{0}},
$$

where the first equation is due to the lack of correlation in the data sequence, as well as the relationship of $(\mathrm{MSE})_{o}=\sigma_{c}^{2}(1-$ $\left.U_{0}\right)$ [22], and SINR $=\frac{\sigma_{c}^{2}-(\mathrm{MSE})_{o}}{(\mathrm{MSE})_{o}}$ is the SNR of the DFEMMSE without utilizing the direct path knowledge $\left\{y_{S, k}\right\}$. Given the direct path signal as in (1), $\frac{\sigma_{c}^{2}\left|\alpha_{S, D}\right|^{2}}{2 \mathcal{N}_{0}}$ is the signal-tonoise ratio in $y_{S, k}$. Therefore, the joint DFE-MMSE estimation 
of the data sequence as proposed here achieves the sum of the SNR from the two channels at the input to the slicer. One channel is from the relays in $\mathcal{D}$, and the other one is from the source $N_{S}$ directly. Clearly, as the number of relays increases, the performance improvement expected by also processing the signal from the source, which only contributes a single degree of diversity, decreases due to the diminishing marginal returns for an additional degree of diversity as the diversity order increases.

2) Diversity Gain: As a case study, this section considers a scenario where the number of participating relays is two. This is always the case for the CRP protocol, but could also occur in the ARP protocol when $K=2$. This analysis will demonstrate that the artificial delays introduced in protocol variant $\mathrm{AD}$ are needed to achieve a diversity gain equal to the number of transmitting nodes, which in this scenario is three.

Note the outage probability considered in this paper is defined as the probability that the frame error probability conditioned on the channel fading parameters is below a prescribed threshold [22]. This is equivalent to the probability that the SINR at the input of the slicer of DFE-MMSE is below a resulting threshold $\gamma_{T}$, i.e. $P_{\text {out }} \triangleq \operatorname{Pr}\left[\operatorname{SINR}<\gamma_{T}\right]$.

From Eq. (11), we can see that SINR is essentially the SNR of an unbiased DFE-MMSE [23] for the channel between relays and destination. On the other hand, the fractional spaced equalizer realizes both the matched filter and the symbol rate feed-forward equalizer [24, pp. 388] whose mean squared error satisfies [23]:

$$
\log \left[\frac{\sigma_{c}^{2}}{(\mathrm{MSE})_{o}}\right]=\frac{1}{2 \pi} \int_{-\pi}^{\pi} \log \left(1+\rho\left|S_{h h}(\theta)\right|^{2}\right) d \theta,
$$

where $\rho=\sigma_{c}^{2} / 2 \mathcal{N}_{0} \quad$ and $\quad\left|S_{h h}(\theta)\right|^{2}=$ $\frac{1}{T} \sum_{m}|F(\theta+2 \pi m) / 2 \pi T|^{2}$ is the folded power spectrum of an equivalent composite channel impulse response including both the transmit filter $h_{T x}(t)$ and channel filter $h_{c, R}(t)$ defined in Section III-A. Let $H_{R C}(f)$ and $H_{c, R}(f)$ denote the frequency response of $h_{R C}(t)$ and $h_{c, R}(t)$, respectively. Then we have $F(f)=H_{R C}(f) H_{c, R}(f)$ since the receiver's front end filter has a squared-root-raisedcosine impulse response. For the purpose of simplicity in derivation and without loss of generality, assume the roll-off factor is $\beta=0$. It is straightforward to show that $\left|S_{h h}(\theta)\right|^{2}=\frac{1}{T}|F(\theta / 2 \pi T)|^{2}=T\left|\sum_{i \in \mathcal{D}_{\mathcal{R}}(s)} \alpha_{i, D} e^{-j \tau_{i} \theta / T}\right|^{2}$, for $\theta \in[-\pi, \pi]$.

Denote $\quad C_{1}=\frac{1}{2 \pi} \int_{-\pi}^{\pi} \log \left(1+\rho\left|S_{h h}(\theta)\right|^{2}\right) d \theta$ and $C_{2}=\log \left(1+\rho\left|\alpha_{S, D}\right|^{2}\right)$. It can be shown (see Appendix B) that $P_{\text {out }} \in\left[P_{\text {out }, L}, P_{\text {out }, U}\right]$, where $P_{\text {out }, U}=\operatorname{Pr}\left[C_{1}+C_{2}<2 \log \left(1+\gamma_{T} / 2\right)\right]$ and $P_{\text {out }, L}=\operatorname{Pr}\left[C_{1}+C_{2}<\log \left(1+\gamma_{T}\right)\right]$. Actually, $\left(C_{1}+C_{2}\right) / 2$ is the instantaneous mutual information of a relay channel with relay nodes using an identical codebook independent of what is sent by the source node [25]. It has been shown in [26] that when two relay nodes both have successfully decoded the source message and the relative delay satisfies $\left|\delta_{\tau}\right| B_{w}>2$, where $\delta_{\tau}=\tau_{1}-\tau_{2}$ and $B_{w}=1 / T$ is the bandwidth of the baseband signal when $\beta=0$, a relay channel having mutual information $\left(C_{1}+C_{2}\right) / 2$ achieves diversity of order 3 , i.e.

$$
\frac{-\log \operatorname{Pr}\left[\left(C_{1}+C_{2}\right) / 2<R\right]}{\log \rho} \rightarrow 3, \text { as } \rho \rightarrow \infty,
$$

where $R$ is a fixed code rate. Thus, under a given SNR threshold $\gamma_{T}$ and as SNR increases, the decoding set almost surely consists of two relay nodes, and it can be concluded that the diversity order defined by $d_{0} \triangleq \lim _{\rho \rightarrow \infty}-\log P_{\text {out }} / \log \rho$ is three based on the bounds of $P_{\text {out }}$ as provided above, as well as Eq. (13).

Therefore, in order to achieve the same diversity order as the synchronous space-time coded approach, the cumulative delay of the disparate paths needs to satisfy the condition of $\left|\delta_{\tau}\right| B_{w}>2$. This implies that there are certain node geometries under which systems employing relays that do not introduce artificial delay (ND) will experience a loss in diversity, hence motivating the use of the Artificial Delay (AD) variant.

\section{Simulation Results}

\section{A. Assumptions}

A network of $K+2$ uniformly distributed nodes in a square area with one side length $D_{L}$ as in Fig. 1 is considered. The maximum propagation delay $T_{D}$ in this area along the diagonal line is set as a rational number of symbol periods $T$, e.g., $T_{D}=3 T$. If the bit rate is $2 / T=2(\mathrm{Mbps})$, this translates to an area of one side length $D_{L}=636.5(\mathrm{~m})$. The signalto-noise ratio (SNR) defined as the ratio of the transmitted signal power to the receiver noise power at each transmitter side is $\sigma_{I}^{2} / \mathcal{N}_{0}=1 / \mathcal{N}_{0}$ due to the normalized signal power. Each packet consists of $L_{P}=200$ uncoded QPSK symbols, where $I_{k}=a_{k}+j b_{k}, a_{k}, b_{k} \in\{1,-1\}$. The SRRC transmit filter $h_{T x}(t)$ is truncated to $[-4 T, 4 T]$ with a roll-off factor $\beta=0.35$. The length $L_{b}$ of the FBF is assumed to be equal to the length of the channel, and $L_{f}$ is chose to be equal to twice the channel pre-cursor length. The frame error rate at node $R_{j}$ is $P_{w}^{\left(R_{j}\right)}=1-\left(1-P_{b}^{(j)}\right)^{2 L_{P}}$, where the bit error rate $P_{b}^{(j)}=Q\left[\sqrt{\left|\alpha_{S, R_{j}}\right|^{2} / \mathcal{N}_{0}}\right]$ [20], $Q(x)=\int_{x}^{\infty} \frac{1}{\sqrt{2 \pi}} e^{-x^{2} / 2} d x$. At the node $N_{R_{j}}$, a binary random variable $\theta_{j} \in\{0,1\}$ is generated with probability distribution function $P\left[\theta_{j}=0\right]=$ $P_{w}^{\left(R_{j}\right)}$. The relay node $N_{R_{j}}$ is in the set $\mathcal{D}$ if $N_{R_{j}}$ is one of the possible relay nodes and $\theta_{j}=1$.

For the given channel realization (i.e., given set $\mathcal{D}$, the given realization of all the fading gains), a Gaussian approximation can be employed to calculate the receiver biterror probability for the joint MMSE detection if the data modulation scheme is QPSK; thus, $P_{b} \approx Q[\sqrt{\mathrm{SINR}}]$ [27]. For a packet of $L_{P}$ QPSK symbols, the instantaneous packet error rate $P_{w}$ is then estimated as $P_{w}=1-\left(1-P_{b}\right)^{2 L_{P}}$ [27]. At the destination, the matched filter bound can serve as a benchmark for the instantaneous BER [28], which is $P_{b, m f}=Q\left[\sqrt{E_{s, m f} / \mathcal{N}_{0}}\right]$. The quantity $E_{s, m f}$ is the joint signal power from the direct path and the path from multiple relays in $\mathcal{D}$ without considering the impact of ISI, and is given by $E_{s, m f}=\int_{-\infty}^{\infty}\left|h_{D, T}(t)\right|^{2} d t+\left|\alpha_{S, D}\right|^{2}$, where $h_{D, T}(t)=$ $\sum_{i \in \mathcal{D}} \alpha_{i, D} h_{T x}\left(t-\tau_{i}\right)$. Thus the packet error rate using the matched filter bound is $P_{w, m f}=1-\left(1-P_{b, m f}\right)^{2 L_{P}}$. The 


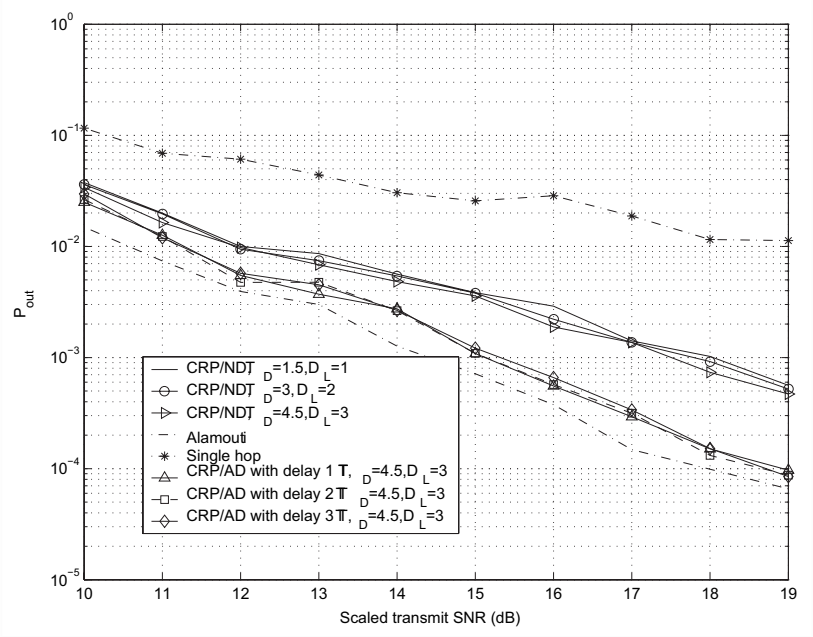

Fig. 4. Outage probability comparison of Protocol CRP with that of [4] employing an Alamouti code. The path loss coefficient $\mu=3$. The number of nodes in an area is $K+2=100 . D_{L}$ is the length of one side of the square region considered. $T_{D}$ is the maximum propagation delay in the region in terms of the number of symbol periods.

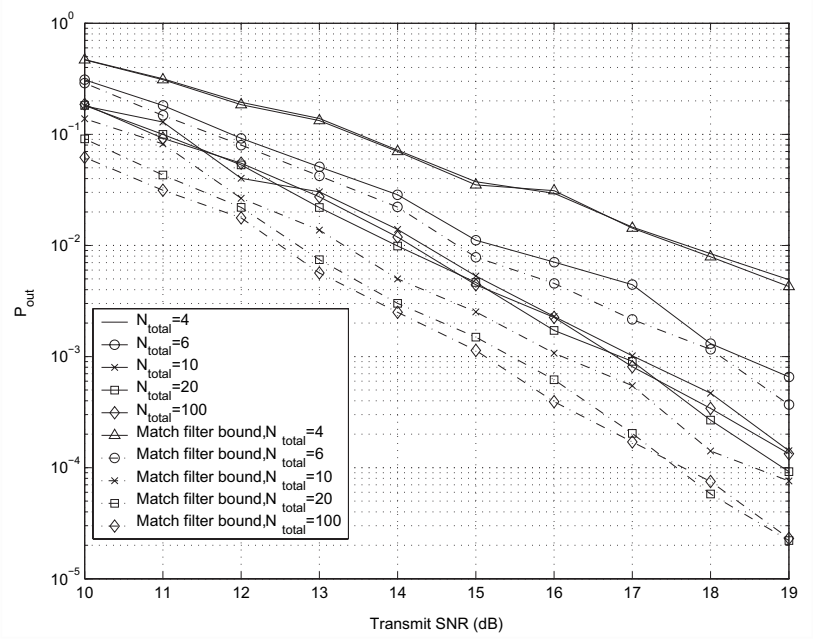

Fig. 5. The outage probability of Protocol ARP/ND with normalization of the received noise power. In the simulations, the parameters are set as $\mu=0$, $D_{L}=3, T_{D}=4.5 T$.

threshold $P_{o}$ under which to declare an outage event is set to 0.1 in the simulation results.

\section{B. Results}

The performance of the proposed schemes will be compared with that of [4] in which an orthogonal space-time block code [29] is employed under the assumption that the relay symbol boundaries are synchronized at the destination. This comparison allows the determination of how much performance can be retained by the proposed protocols while relaxing the symbol synchronization requirements.

In Fig. 4, the outage probability of the CRP protocol is compared with that of [4] employing the space-time orthogonal block codes when both of the two nearest nodes are available to forward the correctly received packets. The Alamouti code [29] is employed to serve this space-time block coding purpose by assuming symbol synchronization. Also considered for comparison is the single hop transmission without any relaying as indicated by the dotted-star line in Fig. 4, which is allowed to increase its transmit power proportionally if $M$ is larger than zero in the relaying schemes, i.e., the transmit SNR is $(1+M) / \mathcal{N}_{0}$, to make a fair comparison. In order to see the impact of relative delays on the performance, $D_{L}$ is increased from 1 to 3 with the maximum delay $T_{D}$ scaled proportionally from $1.5 T$ to $4.5 T$ while fixing $K+2=100$. Since the received SNR at $N_{j}$ from $N_{i}$ is $E\left[\frac{\left|\alpha_{i, j}\right|^{2}}{\mathcal{N}_{0}}\right]=1 /\left(\mathcal{N}_{0} d_{i, j}^{\mu}\right)$, the transmit power will be scaled from 1 to $D_{L}^{\mu}$ to make the average received SNR be $\left(\frac{D_{L}}{d_{i, j}}\right)^{\mu} / \mathcal{N}_{0}$. Therefore, the x-label of Fig. 4 is the average scaled transmit SNR, $D_{L}^{\mu} / \mathcal{N}_{0}$.

From Fig. 4, it can be seen that the CRP protocol using the DFE-MMSE at $N_{D}$ can achieve a diversity gain over the single-hop scheme, as expected. More importantly, compared to [4] using the Alamouti code, there is nearly $3.5 \mathrm{~dB}$ loss for high SNR without introducing any intentional delays (i.e. Variant ND) at the relay nodes. However, for the CRP/AD protocol, the result demonstrates that the performance is only slightly $(<1 \mathrm{~dB})$ worse than a synchronous system. Therefore, it is concluded that even if symbol synchronization is impossible due to the infrastructureless nature of an ad hoc wireless network, comparable performance can be obtained by employing the decision feedback equalizer at $N_{D}$ and setting the delays as a new resource for the involved relay nodes to compete. Increasing the maximum delay $T_{D}$ from $1.5 T$ to $4.5 T$ does not affect the gain much under variant ND, which is expected since in the CRP protocol only nearest two nodes are selected leading to irresolvability of two paths when no extra delay is inserted as in variant $\mathrm{AD}$. Also notice even under variant $\mathrm{AD}$, there is also not much performance improvement when the intentional delay is increased from $1 T$ to $3 T$. This is because the diversity won't increase if the relative delay between these two paths is beyond a certain value as suggested by the analysis in Section III-B.2.

Fig. 5 demonstrates the performance of the ARP/ND protocol. In Fig. 5 and the following two figures, the path loss exponent is set to zero so that the diversity achieved can be observed more easily. Such a setting would perfectly match a system with some form of closed loop relay power control. However, if such is not performed (or it is not accurate), the same diversity will be achieved in each of the schemes but the performance will be worse. Energy normalization across the whole network is considered in Fig. 5 to show the performance contributed purely by the cooperative diversity gain. With the path loss coefficient $\mu=0$, if $M$ nodes are involved in relaying, the total signal power collected from all these paths plus the one from the source directly is $(M+1)$. Then multiply $\mathcal{N}_{0}$ by a factor of $(M+1) / 3$ if $M \geq 2$ such that the transmit SNR for each node in $\mathcal{D}$ is $\frac{3}{(M+1) \mathcal{N}_{0}}$, where the coefficient 3 is for the purpose of comparing with the case of $K=2$, in which the maximum number of transmit nodes is 3. (one is $N_{S}$, another 2 are relay nodes). In terms of both simulation results and the matched filter bound, it can be observed that as $K+2$ is increased from 10 to 100 in a given area, not much improvement is made on the system performance. This is due to the increasing density of relay 


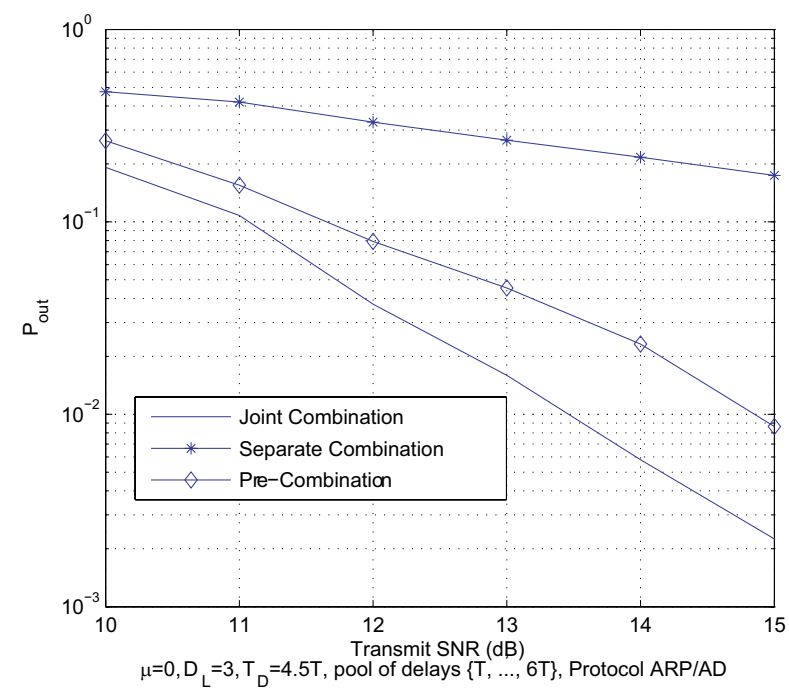

Fig. 6. Outage probability comparison of our proposed joint DFE-MMSE receiver with two other approaches under Protocol ARP/AD. The channel configurations remain the same as in Fig. 7.

nodes in this area, which leads to different clusters of nodes. The delays from $N_{S}$ to the relay nodes in each cluster and then to $N_{D}$ are similar. The number of clusters will determine the asymptotic diversity gain in a given area when equalizer is employed at $N_{D}$.

Fig. 7 demonstrates that for the ARP/AD protocol, randomly introducing the artificial delays to the signals transmitted by the active relay nodes can change the asymptotic tendency observed in Fig. 5 even in the relatively low transmit SNR region, which implies the improvement in diversity gain even if the density of nodes is increased. Note that this protocol requires no coordination of the relays whatsoever, and that the performance will be improved even further by the simple MAC of Section II-B. Hence, it is very suitable for implementation to achieve the gains promised by cooperative diversity. Of course, eventually there are diminishing returns when more relay nodes are added, since the fixed number of resolvable paths for a given delay pool are all exploited with very high probability for any large number of nodes.

Finally, Fig. 6 demonstrates the performance improvement of our proposed joint fractional spaced DFE-MMSE receiver over two other non-trivial approaches using the same system parameters as in Fig. 7. The separate combination scheme refers to an approach in which the coefficient $\beta$ in Fig. 3 is first replaced by the MMSE coefficient for a direct link, i.e. $\hat{\beta}_{0}=\alpha_{S, D}^{*} /\left(\left|\alpha_{S, D}\right|^{2}+2 \mathcal{N}_{0} / \sigma_{c}^{2}\right)$, and the FFF and FBF coefficients are determined assuming $\alpha_{S, D}=0$ in Eq. (4) and Eq. (6). To normalize the linear combination, a scaling factor $1 / 2$ is introduced to both $\hat{\beta}_{0}$ and FFF and FBF coefficients. The pre-combination scheme refers to an approach in which a matched filter is put at the front end of receivers for each link and then the two outputs are added together before performing a fractional spaced DFE-MMSE equalization. The FFF and FBF coefficients in this case can be calculated similarly as in Section III-A. Our simulation results exhibit significant amount of gains over both of these two approaches.

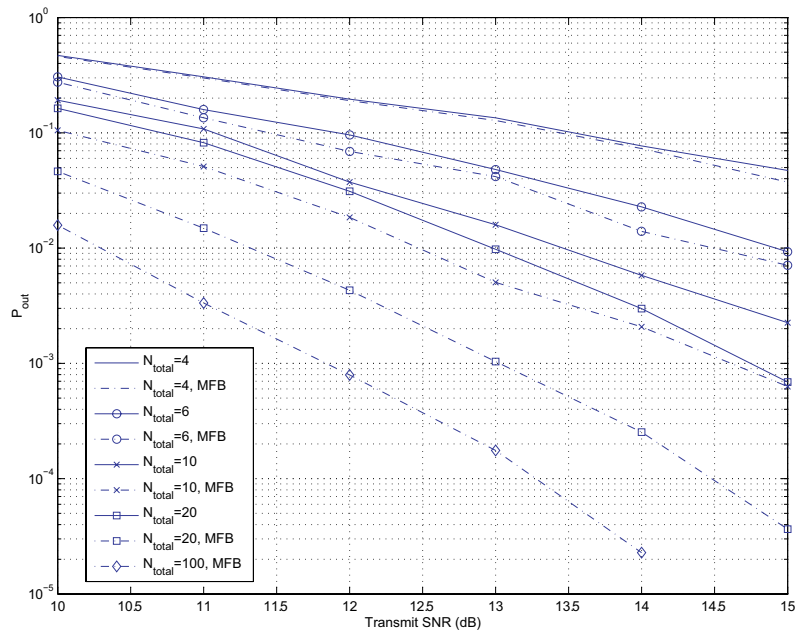

Fig. 7. The outage probability of Protocol ARP/AD with normalization of the received noise power. In simulations, the parameters are set as $\mu=0$, $D_{L}=3, T_{D}=4.5 T$. The pool of delays is $\{T, 2 T, \cdots, 6 T\}$ from which delays are randomly allocated to the packets transmitted by the active relays. MFB in the figure stands for matched filter bound.

\section{COnclusions And Future Work}

In this paper, two delay diversity protocols are proposed to achieve the cooperative diversity gain in an ad hoc wireless network without requiring synchronization of the relayed symbols at the destination. The physical layer approach of employing the DFE-MMSE at the destination was taken as a means to realize such diversity gains, and a novel joint DFE-MMSE equalizer was derived. Based on the proposed outage probability criterion, simulation results demonstrate the performance improvements of the protocols over the single hop scheme, as well as performance comparable to protocols requiring strict symbol synchronization [4]. The result is a promising scheme for realizing the gains of cooperative diversity in networks, without significant signaling overhead or centralized control.

Due to the space constraints, we restrict our attention to two hop relay networks where the destination jointly process the direct link and the relay link information. Our intention is to explore the two-hop case to demonstrate how diversity gain can still be maintained even without symbol level synchronization in wireless networks using our proposed protocols, as well as the novel DFE-MMSE receiver. There are several important issues not addressed in this paper and we give a brief discussion of them below.

The first one is the extension of our work to the multi-hop case. If the outage probability at the destination is too high to be acceptable under a given transmission power constraint, relaying with multiple hops should be deployed. The idea will be similar as the two-hop case. One possible hopping strategy could be that each level of relay nodes retrieves the source information by jointly processing the signals from the two closest previous levels of relay nodes using a joint DFE-MMSE receiver that is similar to the one developed in this paper, or, of course, an analogous joint DFE-MMSE receiver could be derived to process signals from more levels of relaying. The decoding set at each level is subject to both 
the SINR at the input of the slicer under the ARP protocol and locations of these nodes. Incorporating location information is to avoid flooding the whole network in selecting relay nodes [30].

The second is the relationship between the diversity gain and density and geometry of intermediate nodes in a network. Under the ARP protocol, any node which succeeds in decoding the source packets forwards that information towards the destination. In a dense ad-hoc network, the same level of relay nodes could be very close to each other, which makes their transmissions unresolvable even after introducing some random delays from a fixed delay pool as under protocol variant $\mathrm{AD}$. Therefore, achievable diversity gain should be a function of node density and their relative locations, as well as the size of the random delay pool. Investigation of these problems is beyond the scope of this work and will be considered in our future work.

\section{Acknowledgment}

The authors would like to thank Dr. Ananthaswamy Ganesh at Analog Device for his constructive suggestions on the implementations of the DFE-MMSE.

\section{APPENDIX}

\section{A. Derivations of results in Section III}

To determine the vector of coefficients of (3) under the MMSE criterion, the orthogonality principle is employed [20], i.e., $E\left[\left(I_{k}-z_{k}\right) \mathbf{u}_{k}^{*}\right]=0$, where $z_{k}=\mathbf{u}_{k}^{T} \mathbf{c}$, and $\mathbf{u}$ is defined in (2) by assuming the past decisions are error free, $\hat{I}_{k-j}=$ $I_{k-j}, j=1, \cdots, L_{b}$. This yields $E\left[I_{k} \mathbf{u}_{k}^{*}\right]=E\left[\mathbf{u}_{k}^{*} \mathbf{u}_{k}^{T}\right] \mathbf{c}$, and the coefficient vector $\mathbf{c}$ is then given by $\mathbf{c}=\mathbf{R}^{-1} \mathbf{q}$, where $\mathbf{R}=\frac{1}{2} E\left[\mathbf{u}_{k}^{*} \mathbf{u}_{k}^{T}\right]$ is assumed to be a non-singular $\left(L_{f}+L_{b}+\right.$ $1) \times\left(L_{f}+L_{b}+1\right)$ matrix, and $\mathbf{q}=\frac{1}{2} E\left[I_{k} \mathbf{u}_{k}^{*}\right]$ is a column vector of length $L_{f}+L_{b}+1$. Since $\frac{1}{2} E\left[I_{k} I_{j}^{*}\right]=\delta_{i, j}$, where $\delta_{i, j}$ is the Dirac function, it can be shown that

$$
\begin{aligned}
\mathbf{q}= & B\left[h_{D,\left(1-L_{f}\right) / 2}^{*}, \cdots, h_{D,-1}^{*}, h_{D,-1 / 2}^{*}, h_{D, 0}^{*},\right. \\
& \left.\alpha_{S, D}^{*}, \underline{0}\right]^{T}=\left[\mathbf{p}^{T}, \alpha_{S, D}^{*}, \underline{0}\right]^{T},
\end{aligned}
$$

where $\underline{0}$ is a zero row vector of length $L_{b}$. Let $\mathbf{u}_{k}=\left[\mathbf{y}_{D, k}, y_{s, k}, \mathbf{I}_{k-1}\right]^{T}$, where $\mathbf{y}_{D, k}=\left[y_{D, k+\left(L_{f}-1\right) / 2}\right.$, $\left.\cdots, y_{D, k+1}, y_{D, k+1 / 2}, y_{D, k}\right], \quad$ and $\quad \mathbf{I}_{k-1}=$ $\left[I_{k-1}, I_{k-2}, \cdots, I_{k-L_{b}}\right]$. The matrix $\mathbf{R}$ can then be expressed in terms of block sub-matrices:

$$
\mathbf{R}=\left[\begin{array}{cc}
\tilde{\boldsymbol{\Gamma}} & \tilde{\mathbf{\Lambda}}^{*} \\
\tilde{\mathbf{\Lambda}}^{T} & \mathbf{I}_{L_{b}}
\end{array}\right]
$$

where

$$
\begin{aligned}
\tilde{\boldsymbol{\Gamma}} & =\frac{1}{2} E\left(\left[\mathbf{y}_{D, k}, y_{s, k}\right]^{\dagger}\left[\mathbf{y}_{D, k}, y_{s, k}\right]\right) \\
& =\left[\begin{array}{cc}
\boldsymbol{\Gamma} & \alpha_{S, D} \mathbf{p} \\
\alpha_{S, D}^{*} \mathbf{p}^{\dagger} & Q_{0}
\end{array}\right]_{\left(L_{f}+1\right) \times\left(L_{f}+1\right)},
\end{aligned}
$$

$Q_{0}=\frac{1}{2} E\left|y_{S, k}\right|^{2}=\mathcal{N}_{0}+\left|\alpha_{S, D}\right|_{\tilde{\Lambda}}^{2}$, and entries of the matrix $\boldsymbol{\Gamma}$ are defined in (7). The matrix $\tilde{\Lambda}$ is

$$
\tilde{\boldsymbol{\Lambda}}^{*}=\frac{1}{2} E\left(\left[\mathbf{y}_{D, k}, y_{s, k}\right]^{\dagger} \mathbf{I}_{k-1}\right)=\left[\begin{array}{c}
\boldsymbol{\Lambda}^{*} \\
\underline{0}
\end{array}\right]_{\left(L_{f}+1\right) \times\left(L_{b}\right)},
$$

where entries of $\boldsymbol{\Lambda}^{T}$ are defined in (8), and $\mathbf{I}_{L_{b}}$ is a unit matrix of dimension $L_{b} \times L_{b}$.

To determine $\mathbf{c}=\mathbf{R}^{-1} \mathbf{q}$, results for the inversion of a block matrix are needed:

$$
\left[\begin{array}{ll}
\mathbf{A} & \mathbf{B} \\
\mathbf{C} & \mathbf{D}
\end{array}\right]^{-1}=\left[\begin{array}{cc}
\left(\mathbf{A}-\mathbf{B D}^{-1} \mathbf{C}\right)^{-1} & -\mathbf{A}^{-1} \mathbf{B S}_{D}^{-1} \\
-\mathbf{S}_{D}^{-1} \mathbf{C A}^{-1} & \mathbf{S}_{D}^{-1}
\end{array}\right]
$$

where $\mathbf{S}_{D}=\mathbf{D}-\mathbf{C A}^{-1} \mathbf{B}$, and

$$
\begin{aligned}
\left(\mathbf{A}-\mathbf{B D}^{-1} \mathbf{C}\right)^{-1}= & \mathbf{A}^{-1} \mathbf{B}\left(\mathbf{D}-\mathbf{C A}^{-1} \mathbf{B}\right)^{-1} \mathbf{C A}^{-1} \\
& +\mathbf{A}^{-1} .
\end{aligned}
$$

Since the last $L_{b}$ entries of $\mathbf{q}$ are zero, using (17) and (15) yields

$$
\begin{aligned}
{\left[c_{\left(1-L_{f}\right) / 2}, \cdots, c_{-1 / 2}, c_{0}, \beta_{0}\right]^{T}=} & \left(\tilde{\boldsymbol{\Gamma}}-\tilde{\boldsymbol{\Lambda}}^{*} \tilde{\boldsymbol{\Lambda}}^{T}\right)^{-1} \\
& \cdot\left[\mathbf{p}^{T}, \alpha_{S, D}^{*}\right]^{T},
\end{aligned}
$$

where

$$
\begin{aligned}
& \left(\tilde{\boldsymbol{\Gamma}}-\tilde{\boldsymbol{\Lambda}}^{*} \tilde{\boldsymbol{\Lambda}}^{T}\right)^{-1}=\left[\begin{array}{cc}
\boldsymbol{\Gamma}-\boldsymbol{\Lambda}^{*} \boldsymbol{\Lambda}^{T} & \alpha_{S, D} \mathbf{p} \\
\alpha_{S, D}^{*} \mathbf{p}^{\dagger} & Q_{0}
\end{array}\right]^{-1} \\
= & {\left[\begin{array}{cc}
\left(\boldsymbol{\Omega}-\left|\alpha_{S, D}\right|^{2} Q_{0}^{-1} \mathbf{p p}^{\dagger}\right)^{-1} & -\alpha_{S, D} S_{Q}^{-1} \boldsymbol{\Omega}^{-1} \mathbf{p} \\
-\alpha_{S, D}^{*} S_{Q}^{-1} \mathbf{p}^{\dagger} \boldsymbol{\Omega}^{-1} & S_{Q}^{-1}
\end{array}\right], }
\end{aligned}
$$

$S_{Q}=Q_{0}-\left|\alpha_{S, D}\right|^{2} \mathbf{p}^{\dagger} \boldsymbol{\Omega}^{-1} \mathbf{p}$, and $\boldsymbol{\Omega}=\boldsymbol{\Gamma}-\boldsymbol{\Lambda}^{*} \boldsymbol{\Lambda}^{T}$. Based on (19) and (18), it is then straightforward to get (4) and (5).

The input of the slicer is

$$
\begin{gathered}
z_{k}=\mathbf{u}_{k}^{T} \mathbf{c}=\sum_{i=-\left(L_{f}-1\right) / 2}^{0} y_{D, k-i} c_{i}+\sum_{j=1}^{L_{b}} c_{j} I_{k-j}+\beta_{0} y_{S, k} \\
=I_{k}\left(\beta_{0} \alpha_{S, D}+\sum_{i=\left(1-L_{f}\right) / 2}^{0} h_{D,-i} c_{i}\right)+\beta_{0} \nu_{S, k}+\sum_{j=1}^{L_{b}} c_{j} I_{k-j} \\
+\sum_{l \neq 0} I_{k-l}\left(\sum_{i=\left(1-L_{f}\right) / 2}^{0} h_{D, l-i} c_{i}\right)+\sum_{i=\left(1-L_{f}\right) / 2}^{0} c_{i} \nu_{D, k-i} \\
=I_{k} U_{0}+\sum_{n \neq 0, \text { int }} I_{k-n} U_{n}+\tilde{\nu}_{k},
\end{gathered}
$$

hence, in order to cancel the post-cursor interference (i.e., $\sum_{n>0} I_{k-n} U_{n}$ ), the feedforward coefficients must be set as in (6) for the filter length $L_{b} \geq L$ [20].

For a DFE-MMSE equalizer, the mean squared error can be determined by the coefficient $U_{0}$ in (20), i.e., MSE $=\sigma_{c}^{2}(1-$ $\left.U_{0}\right)$ as shown in [22]. Given $\beta_{0}$ and the FFF coefficients as in (5) and (4), respectively,

$$
\begin{aligned}
U_{0}= & \beta_{0} \alpha_{S, D}+\sum_{i=\left(1-L_{f}\right) / 2}^{0} h_{D,-i} c_{i} \\
= & \mathbf{p}^{\dagger} \boldsymbol{\Omega}^{-1} \mathbf{p}\left(1-\beta_{0} \alpha_{S, D}\right)+\beta_{0} \alpha_{S, D} \\
= & \left|\alpha_{S, D}\right|^{2} \frac{\left(1-\mathbf{p}^{\dagger} \boldsymbol{\Omega}^{-1} \mathbf{p}\right)^{2}}{2 \mathcal{N}_{0} / \sigma_{c}^{2}+\left|\alpha_{S, D}\right|^{2}\left(1-\mathbf{p}^{\dagger} \mathbf{\Omega}^{-1} \mathbf{p}\right)} \\
& +\mathbf{p}^{\dagger} \boldsymbol{\Omega}^{-1} \mathbf{p} .
\end{aligned}
$$

By substituting (21) in $\sigma_{c}^{2}\left(1-U_{0}\right)$, (9) is obtained.

It has been shown in [22] that the SINR at the input to the slicer is $\sigma_{c}^{2} / \mathrm{MSE}-1$ for any MMSE equalizer. Given 
the minimum MSE in (9) for the joint estimation, it is then straightforward to prove (11).

\section{B. Bounds on Outage Probability $P_{\text {out }}$}

From Eq. (11) and definitions of $C_{1}$ and $C_{2}$ in Section III-B.2, we have SINR $=2^{C_{1}}+2^{C_{2}}-2$. Using inequality $a+b \geq 2 \sqrt{a b}$ for non-negative numbers $a$ and $b$, we obtain SINR $\geq 2^{1+\left(C_{1}+C_{2}\right) / 2}-2$. Therefore, the outage probability $P_{\text {out }}=\operatorname{Pr}\left[\operatorname{SINR}<\gamma_{T}\right]$ is upper bounded by

$$
\begin{aligned}
P_{\text {out }, U} & \triangleq \operatorname{Pr}\left[2^{1+\left(C_{1}+C_{2}\right) / 2}-2<\gamma_{T}\right] \\
& =\operatorname{Pr}\left[C_{1}+C_{2}<2 \log \left(1+\gamma_{T} / 2\right)\right] .
\end{aligned}
$$

Similarly, we need to first find the upper-bound of SINR in order to derive the lower-bound of $P_{\text {out }}$. Let us denote $a_{1}=\rho\left|\alpha_{S, D}\right|^{2}$ and $b_{1}(\theta)=1+\rho\left|S_{h h}(\theta)\right|^{2}$. From Eq. (11) and Eq. (12), we can deduce that

$$
\frac{\sigma_{c}^{2}}{(\mathrm{MSE})_{o}}=2^{\frac{1}{2 \pi} \int_{-\pi}^{\pi} \log \left[b_{1}(\theta)\right] d \theta}+2^{\frac{1}{2 \pi} \int_{-\pi}^{\pi} \log \left(a_{1}\right) d \theta} .
$$

Let $\log Z=\frac{1}{2 \pi} \int_{-\pi}^{\pi} \log \left(a_{1}+b_{1}(\theta)\right) d \theta$, we then obtain the following inequality

$$
\begin{aligned}
& \frac{\sigma_{c}^{2} /(\mathrm{MSE})_{o}}{Z}=2^{\frac{1}{2 \pi} \int_{-\pi}^{\pi} \log \frac{b_{1}(\theta)}{a_{1}+b_{1}(\theta)} d \theta}+2^{\frac{1}{2 \pi} \int_{-\pi}^{\pi} \log \frac{a_{1}}{a_{1}+b_{1}(\theta)} d \theta} \\
& \quad \leq \frac{1}{2 \pi} \int_{-\pi}^{\pi} 2^{\log \frac{b_{1}(\theta)}{a_{1}+b_{1}(\theta)}} d \theta+\frac{1}{2 \pi} \int_{-\pi}^{\pi} 2^{\log \frac{a_{1}}{a_{1}+b_{1}(\theta)}} d \theta \\
& =\frac{1}{2 \pi} \int_{-\pi}^{\pi} \frac{a_{1}+b(\theta)}{a_{1}+b(\theta)} d \theta=1
\end{aligned}
$$

where the inequality is due to the convexity of exponential function. Given the definition of $C_{1}$ and $C_{2}$, we have $\log Z<$ $\log \left(1+a_{1}\right)+\frac{1}{2 \pi} \int_{-\pi}^{\pi} \log b(\theta) d \theta=C_{1}+C_{2}$ which results in

$$
\mathrm{SINR}=\frac{\sigma_{c}^{2}}{(\mathrm{MSE})_{o}}-1<2^{C_{1}+C_{2}}-1 .
$$

The upper-bound of SINR yields the following lower-bound of $P_{\text {out }}$, i.e.

$$
\begin{aligned}
P_{\text {out }} & \geq P_{\text {out }, L} \triangleq \operatorname{Pr}\left[2^{C_{1}+C_{2}}-1<\gamma_{T}\right] \\
& =\operatorname{Pr}\left[C_{1}+C_{2}<\log \left(1+\gamma_{T}\right)\right] .
\end{aligned}
$$

We have thus developed bounds for $P_{\text {out }}$ as defined in Eq. (22) and Eq. (26).

\section{REFERENCES}

[1] A. Goldsmith and S. Wicker, "Design challenges for energy-constrained ad hoc wireless networks," IEEE Personal Commun., vol. 9, pp. 8-22, Aug. 2002.

[2] A. Sendonaris, E. Erkip, and B. Aazhang, "User cooperation diversitypart I: system description," IEEE Trans. Commun, vol. 51, pp. 19271938, Nov. 2003.

[3] J. N. Laneman and G. W. Wornell, "Energy-efficient antenna-sharing and relaying for wireless networks," in Proc. IEEE Wireless Communications and Networking Conference WCNC, Chicago, IL, Sept. 2000.

[4] J. Laneman and G. Wornell, "Distributed space-time coded protocols for exploiting cooperative diversity in wireless networks," IEEE Trans. Inform. Theory, vol. 49, pp. 2415-2425, Oct. 2003.

[5] A. Stefanov and E. Erkip, "Cooperative coding for wireless networks," in Proc. IEEE Conference on Mobile and Wireless Communications Networks, Stockholm, Sweden, Sept. 2002.
[6] T. Hunter and A. Nosratinia, "Coded cooperation under slow fading, fast fading, and power control," in Proc. Asilomar Conference on Signals, Systems, and Computers, Nov. 2002.

[7] A. Stefanov and E. Erkip, "Cooperative space-time coding for wireless networks," in Proc. IEEE Information Theory Workshop, La Sorbonne, Paris, France, April 2003.

[8] A. Scaglione and Y. W. Hong, "Opportunistic large arrays: cooperative transmission in wireless multihop ad hoc networks to reach far distances," IEEE Trans. Signal Proc., vol. 51, pp. 2082-2092, Aug. 2003.

[9] M. Hasna and M.-S. Alouini, "End-to-end performance of transmission systems with relays over Rayleigh-fading channels," IEEE Trans. Wireless Comm., vol. 2, no. 6, pp. 1126- 1131, Nov. 2003.

[10] M. Hasna and M.-S. Alouini, "Harmonic mean and end-to-end performance of transmission systems with relays," IEEE Trans. Commun., vol. 52, no. 1, pp. 130-135, Jan. 2004.

[11] D. T. J. Laneman and G. Wornell, "Cooperative diversity in wireless networks: efficient protocols and outage behavior," IEEE Trans. Inform. Theory, vol. 50, pp. 3062-3080, Dec. 2004.

[12] J. Boyer, D. Falconer, and H. Yanikomeroglu, "Multihop diversity in wireless relaying channels," IEEE Trans. Commun., vol. 52, pp. 18201830, Oct 2004.

[13] D. Goeckel and Y. Hao, "Macroscopic space-time coding: motivation, performance cirteria, and a class of orthogonal designs," in Proc. Conference on Information Sciences and Systems (CISS), March 2003.

[14] N. Seshadri and J. H. Winters, "Two signaling schemes for improving the error performance of frequency-division-duplex (FDD) transmission systems using transmitter antenna diversity," in Proc. 43rd IEEE Vehicular Technology Conference, 1993, pp. 508-511.

[15] S. Lin and D. J. Costello, Error Control Coding: Fundamentals and Applications. Prentice-Hall, 1983.

[16] T. S. Rappaport, Wireless Communications: Principles and Practice. New Jersey: Prentice Hall, Inc., 1996.

[17] M. A. Khojastepour, A. Sabharwal, and B. Aazhang, "On the capacity of 'cheap' relay networks," in Proc. Conference on Information Sciences and Systems (CISS), March 2003.

[18] J. E. Smee and N. C. Beaulieu, "Error-rate evaluation of linear equalization and decision feedback equalization with error propagation," IEEE Trans. Commun., vol. 46, pp. 656-665, May 1998.

[19] I. J. Fevrier, S. Gelfand, and M. Fitz, "Reduced complexity decision feedback equalization for multipath channels with large delay spreads," IEEE Trans. Commun., vol. 47, pp. 927-937, June 1999.

[20] J. G. Proakis, Digital Communications, 3rd ed. New York: McGrawHill, 1995.

[21] J. E. Smee and N. C. Beaulieu, "On the equivalence of the simultaneous and seperate MMSE optimizations of a DFE FFF and FBF," IEEE Trans. Commun., vol. 45, pp. 156-158, 1997.

[22] P. Balaban and J. Salz, "Optimum diversity combining and equalization in digital transmission with applications to celluar mobile radio - part I \& II,", IEEE Trans. Commun., vol. 40, pp. 885-907, May 1992.

[23] J. M. Cioffi, G. P. Dudevoir, M. V. Eyuboglu, and G. D. Forney, "MMSE decision-feedback equalizers and coding II: coding results," IEEE Trans. Commun., vol. 43, pp. 2595-2603, Oct. 1995.

[24] J. R. Barry, E. A. Lee, and D. G. Messerschmitt, Digital Communications. Kluwer Academic, 2004.

[25] S. Wei, "Diversity-mutlipexing tradeoff of asynchronous space-timecoded cooperative diversity," Proc. Allerton Conf. Commun., Control, Computing, Oct. 2004.

[26] S. Wei, "Diversity-multiplexing tradeoff of asynchronous cooperative diversity in wireless networks," IEEE Trans. Inform. Theory, Feb. 2006 , 1st revision submitted.

[27] S. Ariyavisitabul and L. J. Greenstein, "Reduced complexity equalization techniques for broadband wireless channes," IEEE J. Select. Areas Commun., vol. 15, pp. 5-15, Jan. 1997.

[28] F. Ling, "Matched filter-bound for time-discrete multipath Rayleigh fading channels," IEEE Trans. Commun., vol. 43, pp. 710-713, Feb./March/April 1995.

[29] S. M. Alamouti, "A simple transmit diversity technique for wireless communications," IEEE J. Select. Areas Commun., vol. 16, pp. 14511457, Oct. 1998.

[30] B. Sirkeci-Mergen and A. Scaglione, "A continuum approach to dense wireless networks with cooperation," in IEEE Infocom, Miami, U.S., March 13-17, 2005. 


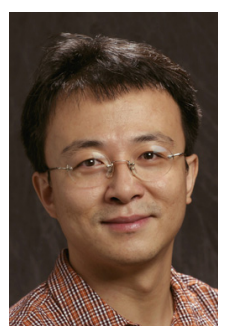

Shuangqing Wei graduated in Electrical Engineering from Tsinghua University with BE and MS in 1995 and 1998 and then obtained his Ph.D in EE from the University of Massachusetts, Amherst in 2003. He is currently an Assistant Professor in the EE department at Louisiana State University. His areas of interest are in communication theory, information theory, coding theory and their applications to wireless networks.

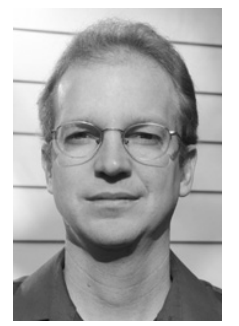

Dennis L. Goeckel split time between Purdue University and Sundstrand Corporation from 19871992, receiving his BSEE from Purdue in 1992. From 1992-1996, he was a National Science Foundation Graduate Fellow and then Rackham PreDoctoral Fellow at the University of Michigan, where he received his MSEE in 1993 and his Ph.D. in 1996, both in Electrical Engineering with a specialty in Communication Systems. In September 1996, he joined the Electrical and Computer Engineering department at the University of Massachusetts, where he is currently an Associate Professor. His current research interests are in the areas of signal processing and communication theory.

Dr. Goeckel was the recipient of a 1999 CAREER Award from the National Science Foundation for "Coded Modulation for High-Speed Wireless Communications". He was a Lilly Teaching Fellow at UMass-Amherst for the 2000-2001 academic year. He served as an Editor for the IEEE Journal on Selected Areas in Communications: Wireless Communication Series during its transition to the IEEE Transactions on Wireless Communications from 1999-2002, and as a Technical Program Committee Co-Chair for the Communication Theory Symposium at Globecom 2004. He is currently an Editor for the IEEE Transactions on Communications.

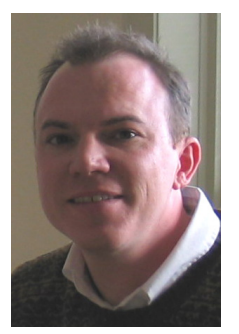

Matthew C. Valenti received a B.S.E.E. from Virginia Tech, Blacksburg, in 1992, a M.S.E.E. from the Johns Hopkins University, Baltimore, MD, in 1995, and a Ph.D. in electrical engineering from Virginia Tech, in 1999, where he was a Bradley Fellow. He is currently an Associate Professor in the Lane Department of Computer Science and Electrical Engineering at West Virginia University, Morgantown. He serves as an associate editor for IEEE Transactions on Vehicular Technology, and has been on the technical program committee for several international conferences. His research interests are in the areas of communication theory, error correction coding, applied information theory, and wireless multiple-access networks. He also acts as a consultant to several companies engaged in various aspects of turbo codec design, including software radio, FPGA, and ASIC implementations for military, satellite, and third generation cellular applications. Prior to attending graduate school at Virginia Tech, he was an electronics engineer at the United States Naval Research Laboratory, Washington, DC. 